\title{
Multimodal encoding in a simplified model of intracellular calcium signaling
}

\author{
Maurizio De Pittà • Vladislav Volman • \\ Herbert Levine $\cdot$ Eshel Ben-Jacob
}

Published online: 10 January 2009

(C) Marta Olivetti Belardinelli and Springer-Verlag 2008

\section{Erratum to: Cogn Process}

$$
\text { DOI 10.1007/s10339-008-0242-y }
$$

The legend of Fig. 1 contains errors. Please find below Fig. 1 with the correct figure legend.
(A)
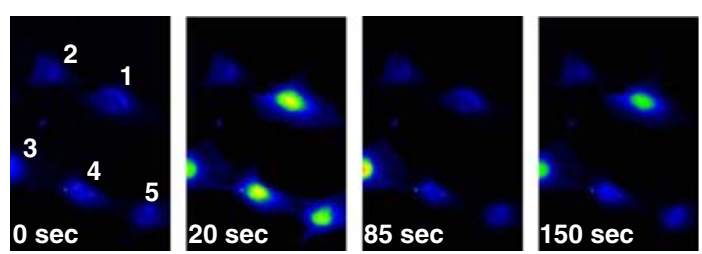

(B)

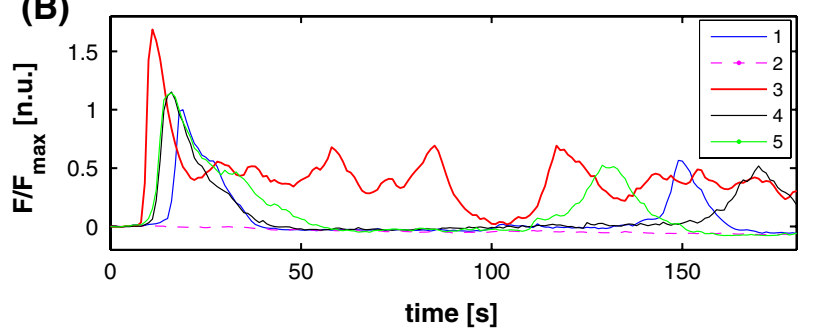

Fig. 1 a Purified astrocytes from rat's visual cortex loaded with calcium indicator fluo-3 and stimulated by norepinephrine $(25 \mu \mathrm{M})$ and (b) associated normalized $\mathrm{Ca}^{2+}$ traces. Similarly to astrocytes, many other cells display intracellular $\mathrm{Ca}^{2+}$ oscillations in response to several external agents. Such oscillations are characterized by great variability both in their peaks and in their frequency, hinting complex modes of encoding of information of external signals. Images and experimental data courtesy of V. Parpura [adapted from: Lee W, Parpura V (2007) Exocytotic release of glutamate from astrocytes: comparison to neurons. In: Bean A (ed) Protein trafficking in neurons. Elsevier, Amsterdam, pp 329-365]

School of Physics and Astronomy, Tel Aviv University, 69978 Ramat Aviv, Israel

e-mail: eshel@tamar.tau.ac.il

V. Volman · H. Levine · E. Ben-Jacob

Center for Theoretical Biological Physics,

UCSD, La Jolla, CA 92093-0319, USA

\section{Volman}

Computational Neurobiology Lab, The Salk Institute,

La Jolla, CA 92037, USA 